Musées, Patrimoine et Culture scientifiques et techniques

$190 \mid 2020$

juillet-août 2020

\title{
Le muséum, acteur engagé en culture scientifique et technique?
}

\section{Philippe Guillet et Samuel Cordier}

\section{(2) OpenEdition \\ Journals}

Édition électronique

URL : https://journals.openedition.org/ocim/3902

DOI : $10.4000 /$ ocim.3902

ISSN : 2108-646X

Éditeur

OCIM

Édition imprimée

Date de publication : 1 juillet 2020

Pagination : 12-17

ISSN : 0994-1908

Référence électronique

Philippe Guillet et Samuel Cordier, «Le muséum, acteur engagé en culture scientifique et technique

? », La Lettre de I'OCIM [En ligne], 190 | 2020, mis en ligne le 01 juillet 2021, consulté le 17 juillet 2021.

URL : http://journals.openedition.org/ocim/3902 ; DOI : https://doi.org/10.4000/ocim.3902

Ce document a été généré automatiquement le 17 juillet 2021.

Tous droits réservés 


\section{Le muséum, acteur engagé en culture scientifique et technique?}

\section{Philippe Guillet et Samuel Cordier}

Éternité-Rêve humain et réalités de la science (2017), une « exposition d'intérêt national ».

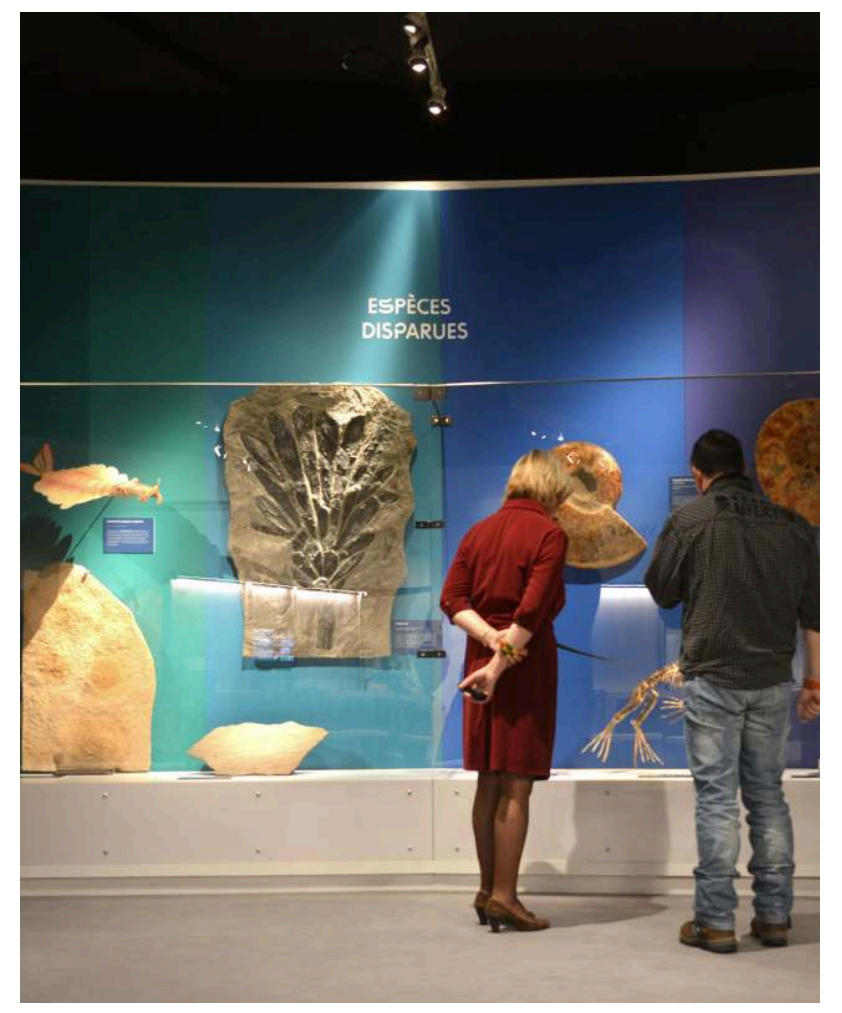

(c) Muséum de Nantes

SAMUEL CORDieR: Au moment de rouvrir le muséum de Nantes au public, quels premiers enseignements de la période de confinement tirez-vous en termes de fonctionnement?

PHILIPPE GUILLET : Je serai assez bref sur ma réponse car elle est commune à de nombreux musées et au bout du compte, assez banale. En termes de fonctionnement du musée, il y 
aura des évolutions, mais pas de façon drastique. La communication entre collègues et professionnels va évoluer. Au cours de la période de confinement, nous avons eu recours aux visioconférences pour communiquer entre nous. Avec nos partenaires, ce mode de communication a bien sûr été aussi amplifié. Et, puis au bout du confinement, nous avons éprouvé la nécessité de se revoir physiquement. Certes, nous continuerons à utiliser les outils qui nous ont accompagnés durant cette période mais de façon plus parcimonieuse, comme se développera le télétravail pour les collaborateurs quand leur tâche le permettra.

sc: Vous avez également maintenu une offre aux publics pendant la période de confinement.

PG : En effet, pendant la période de confinement, nous avons suivi le mouvement et proposé des offres sur notre site web, notamment des nouvelles médiations. La présence du muséum sur Internet a répondu à une demande très forte de la métropole, mais nos propositions ont été probablement très au-delà de ce que le public peut recevoir. Car très vite nous nous sommes rendus compte que - dans ce flot d'informations - nous avons été peu visibles, juste un succès d'estime: par exemple, quelques centaines de vues pour la visite virtuelle du muséum par un drone. Nous ne rivaliserons jamais avec d'autres sources dans le domaine des sciences et de sa vulgarisation. Si Jamy Gourmaud touche d'emblée plusieurs centaines de milliers de personnes, nous arrivons sur certaines de nos mises en ligne à atteindre péniblement 100 ou 150 personnes. Est-ce que cela vaut le coup d'investir en temps et en moyens sur certains sujets et mimer ce qui est fait par ailleurs en beaucoup mieux?

Ce questionnement n'est pas nouveau car c'est le même qui se pose sur les thématiques d'expositions. Faut-il un documentaire de 52 minutes, un nouvel ouvrage, une nouvelle appli ? Quelle est notre force par rapport aux autres médias ? Les collections bien entendu ! Retour aux fondamentaux du musée, c'est ce sur quoi nous devons prioritairement bâtir nos médiations.

$\boldsymbol{s c}$ : Selon vous, pendant cette période de confinement et aujourd'hui encore, le rôle et l'importance des collectivités territoriales n'ont pas été suffisamment mentionnés?

PG : Effectivement, quand j'ai lu les articles de la lettre de l'Ocim nº 189 (mai-juin 2020) pendant le confinement, dans la rubrique «Distances », j'ai été très surpris par le fait qu'à aucun moment, le rôle des collectivités territoriales n'ait été évoqué. Or, il ne faut pas oublier que tous les muséums s'insèrent dans une collectivité d'une manière ou d'une autre: pour l'immense majorité, ce sont des services en régie, il y a quelques EPCC et de rares associations. Le directeur du musée est ainsi lui-même dans une position hiérarchique définie dans un organigramme et, selon le fonctionnement de la collectivité, peut participer ou non à l'établissement de la politique publique le concernant.

À Nantes, les directeurs et l'équipe de direction sont impliqués. Mais il existe aussi des collectivités où les directeurs n'ont pas leur mot à dire: ils sont simplement là pour appliquer les décisions des élus en lien avec le directeur général de la culture. Je crois que c'est une condition aux limites qu'il faut avoir à l'esprit lorsque nous parlons d'engagement du musée. J'ai en mémoire un directeur général des services d'une grande collectivité qui, un peu par provocation, n'hésitait pas à me dire : «la biodiversité, je m'en fiche. Mon objectif est de créer plusieurs milliers de logements car nous en avons un besoin urgent ». Le plus beau de nos discours ne servait à rien pour le convaincre. Ce qui me permet de dire ici que nous avons un rôle de formation des élus et des directeurs généraux sur les questions de culture scientifique, il faut s'appuyer sur nos associations et d'autres instances comme I'IHEST qui s'y emploie. 
Rendre tangibles les émotions en créant le lien avec l'objet. Galerie de zoologie.

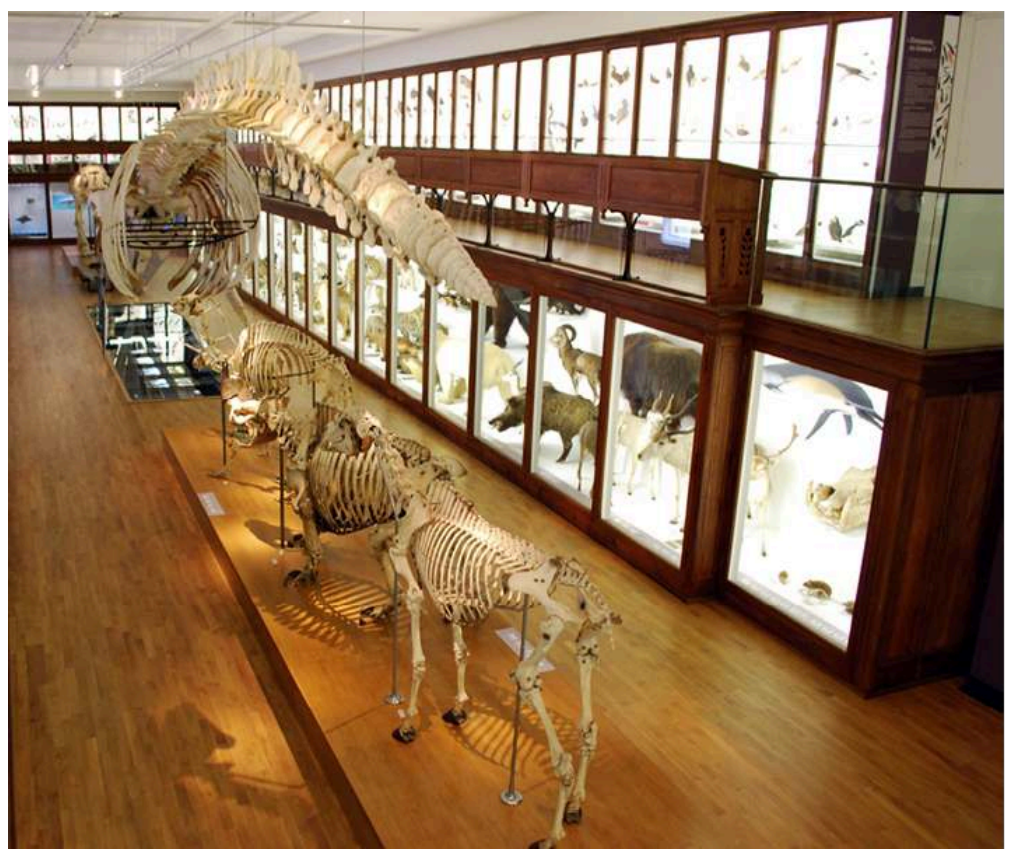

(c) Muséum de Nantes

$\boldsymbol{s c}$ : La révolution annoncée au sein de la sphère muséale serait donc à nuancer?

PG : Par rapport à ce que j'ai entendu ou lu, lié au projet de révolutionner le monde d'après, il faut en effet s'entendre. Car, positionnés hiérarchiquement, les directeurs d'une institution muséale sont contraints. Nous ne sommes pas libres de faire ou dire ce que l'on veut. Nous devons en référer pour tout sujet d'ordre politique au cabinet de l'élu ou à notre hiérarchie. Je prends un exemple très récent lié à la question du racisme et des luttes associées. J'entends dire «le musée est politique»; oui dans la mesure où il s'insère dans un projet politique de la collectivité. Mais le directeur de l'institution ne peut engager sa collectivité.

Par contre, il est du devoir du directeur de proposer et de pouvoir ainsi traiter des thématiques en rapport avec l'actualité, avec ce que le musée peut apporter d'informations solides et vérifiables. C'est un point important qui rejoint effectivement la notion de «musée engagé ». Et pour les muséums, pour ce qui nous concerne, ce sont dans les questions environnementales dont la biodiversité, que le muséum doit s'engager. Dans le contexte actuel, c'est la force du musée de sciences et c'est maintenant que le muséum doit se montrer sur des sujets d'actualité, des sujets forts et de société.

Cette réflexion n'est pas nouvelle, loin s'en faut, et je repense au congrès d'Ungersheim, celui de la fondation de la Fédération des écomusées et musées de société qui a eu lieu en juin 1991. Dans le compte-rendu que j'en avais fait dans la lettre de l'Ocim n 17 (septembreoctobre 1991), je me demandais si le muséum ne pouvait pas être considéré lui aussi comme musée de société. Je citerai aussi comme marqueur de cette histoire l'ouvrage de Jean Davallon, Gérald Grandmont et Bernard Schiele publié en 1993 : L'environnement entre au musée. Enfin je rappellerai que la notion de «musée engagé » avait été abordée lors du congrès de la Société des musées du Québec en 2001, cela fait bientôt 20 ans.

sc : Au sortir de cette crise, la position des muséums - interlocuteurs privilégiés sur les questions sociétales et environnementales - semble renforcée?

PG : En effet, et c'est toujours cette notion d'engagement qui me paraît essentielle. Nous ne sommes plus dans les expos "petites fleurs-petits oiseaux» depuis longtemps, nous sommes passés à une autre étape de notre développement. Notre crédibilité est aussi là. Nous ne pouvons pas être en décalage par rapport aux questions que se posent les 
citoyens tout en leur proposant ce moment d'émerveillement et de plaisir propice à la connaissance qu'est la « délectation », selon une ancienne définition du musée par l'Icom.

Les blockbusters, un moyen de raviver l'intérêt scientifique du grand public ? (Mille milliards de fourmis, 2016).

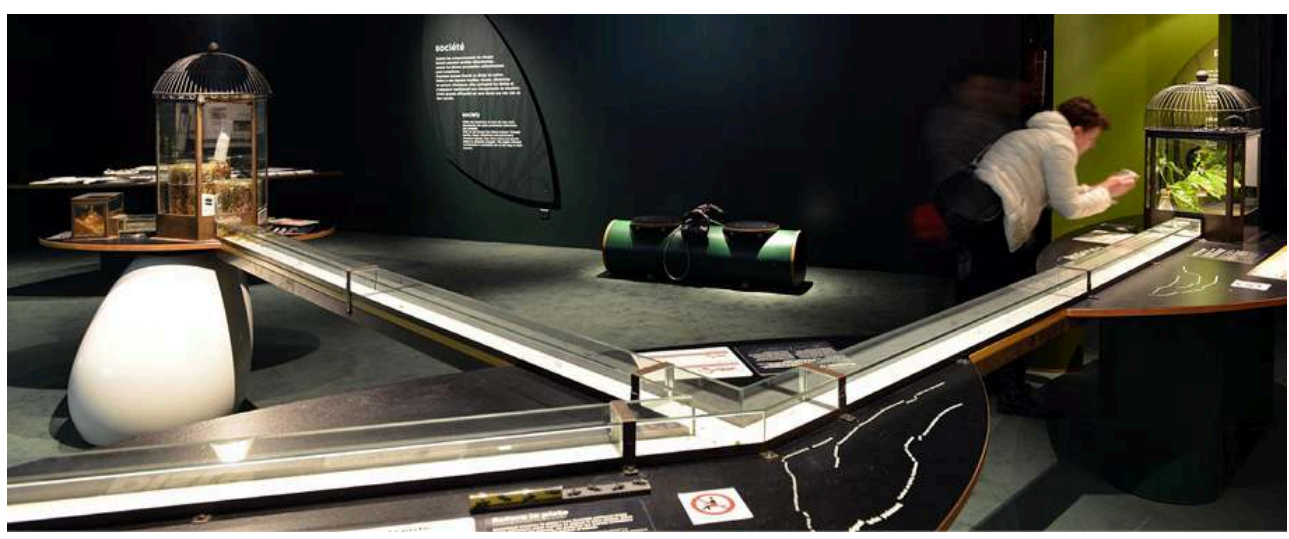

(c) Muséum de Nantes

sc : Et dans cette perspective vous soulignez que le Projet scientifique et culturel (PSC) est une étape essentielle?

PG : Pour moi, le PSC est d'abord un document d'engagement sur lequel vont se prononcer les élus. Et j'avoue avoir beaucoup évolué sur cette question. Lorsque la «Loi musée »a imposé ce document aux Musées de France en 2003, je ne comprenais pas le sens des premiers documents forts de plusieurs centaines de pages sur l'histoire de l'institution et de ses collections absorbant le travail de toute une équipe sur de trop longs mois! Ce n'est heureusement plus le cas, le PSC est maintenant synthétique. II propose une vision et une stratégie développées par le directeur et son équipe souvent en quelques mois et ce PSC est revisité régulièrement. Dès lors, il peut être compris par les édiles qui l'approuvent. Reste ensuite au musée à l'appliquer en développant une feuille de route !

À Nantes, ce document a été soutenu devant le conseil métropolitain en février 2017 et il est devenu une assise pour développer notre engagement en matière d'environnement. Je crois que le muséum est là, pas ailleurs. Dans le débat actuel, nous avons vraiment des connaissances, des informations essentielles à proposer. Dans le domaine de l'environnement, on entend des discours, souvent sans base scientifique (voire avec une base détournée), nous devons donc apporter « ce que la science a à dire » à l'aide de ce qui est notre force première : les collections et notamment les collections anciennes, témoins d'une biodiversité disparue.

sc : Le PSC 2021-2026 pour le muséum de Nantes est en cours d'écriture. Pourquoi vous semble-t-il important de mentionner dans ce document l'ambition du muséum de « répondre à la société actuelle »?

PG : Je cite précisément notre PSC parce que cela me semble important. Dans le PSC 2017-2020, nous avons très clairement annoncé les questions de société et d'environnement: "Conformément aux missions principales qui lui sont dévolues, le muséum de Nantes doit être un lieu porteur de rêves, de découvertes et de créativité, un lieu de rapprochement entre les jeunes et la science, un musée dans son quartier, dans sa ville et dans sa métropole proposant une science du quotidien, une science pour et dans la société, un musée acteur et référent dans les réseaux locaux, nationaux et européens, porteur du rayonnement de la ville de Nantes dans son domaine ».

Les élus se sont prononcés sur ce point et ce document a été validé par le ministère de la Culture, qui lui apporte en quelque sorte son imprimatur.

Nous sommes actuellement à la réécriture du PSC pour la période 2020-2026. Les aspects d'engagement de l'institution, de participation des citoyens dans nos choix y seront 
notoirement plus développés. L'ombre de la période passée pèse fortement sur nos réflexions.

$\boldsymbol{s c}$ : Quelle part de liberté gardent les muséums, notamment en termes de programmation? PG : C'est un point important. Car, si nous n'avons pas cette liberté de choix dans les thématiques et leurs traitements que donne le statut associatif - liberté relative d'ailleurs nous pouvons être mieux entendus par notre collectivité. Et j'ai vu, quand je suis arrivé à Nantes, que des degrés de liberté se gagnaient avant tout par une bonne fréquentation qui découle elle-même d'une bonne programmation. Nous avons présenté des expositions un peu blockbusters, comme Mille milliards de fourmis (2016) ou Au fil des araignées (2018) qui nous ont permis d'accueillir 130 ou 140000 visiteurs. Le cap des 100000 visiteurs est tout à fait psychologique pour nos élus !

Nous gagnons aussi cette liberté, cette crédibilité, grâce à notre volonté d'être très présents sur le territoire et de répondre aux sollicitations dans tous les domaines culturels. Nous ne devons jamais fermer les portes aux partenariats. À partir du moment où le muséum démontre qu'il joue pleinement son rôle d'acteur culturel du territoire, de nouveaux projets et de nouvelles perspectives s'offrent à lui spontanément.

C'est cette crédibilité qui nous a permis de proposer à notre public des thématiques moins évidentes comme "l'éternité » sous un angle un peu plus philosophique. De la même manière, nous préparons une exposition pour 2021 - en partenariat avec l'Espace des sciences de Rennes et le musée du Fjord à Saguenay - sur le thème des intelligences, avec une forte dominante réflexive. Mais la programmation est toujours affaire d'équilibre.

\section{"Nous ne sommes plus dans les expos "petites fleurs-petits oiseaux" depuis longtemps. (...) Nous ne pouvons pas être en décalage par rapport aux questions que se posent les citoyens ". Façade du muséum de Nantes, 2019.}

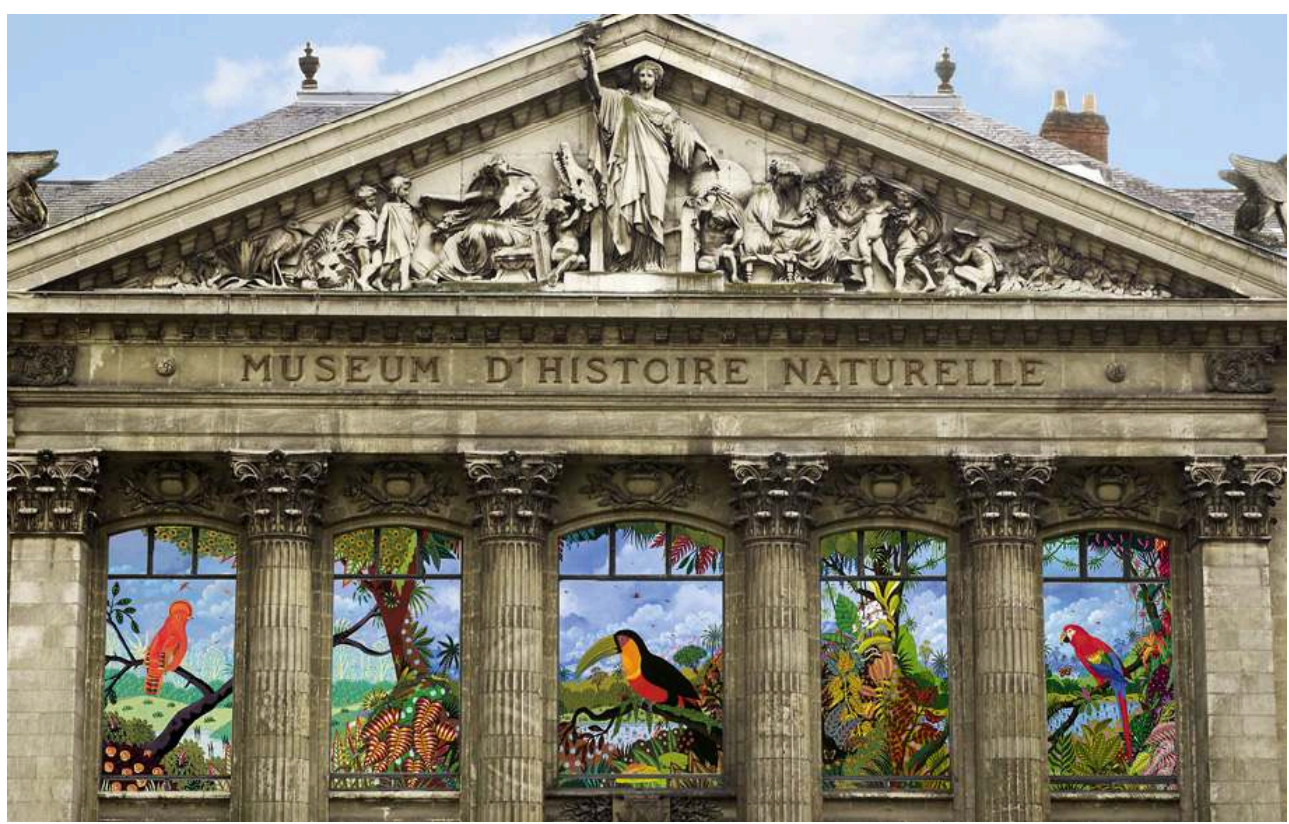

(c) Muséum de Nantes

$\boldsymbol{s c}$ : L'engagement des associations professionnelles vous semble-t-il aussi important dans ce contexte?

PG : Effectivement, et mon expérience à l'Amcsti, à l'Icom ${ }^{1}$ ou à la Conférence permanente des muséums de France (CPMF) m'incite à promouvoir cet engagement auprès des jeunes professionnels. Outre la connaissance que cela apporte sur les champs disciplinaires autres que le sien, sur les institutions et leurs fonctionnements (ce qui peut éventuellement simplifier les demandes de financements), il contribue à renforcer notre crédibilité sur le plan local auprès de nos directeurs généraux et élus qui ne sont pas insensibles à ces 
valeurs d'engagement. S'impliquer dans une association (dans son conseil d'administration par exemple) est certes coûteux en temps et en énergie, mais les retombées pour son établissement vont bien au-delà de cet investissement qui, rappelons-le, a comme but premier de profiter à toute la communauté muséale.

sc: Au cours de la période de confinement, on a vu l'importance du numérique, puis ses limites. Vous aviez participé il y a quelques années à une réflexion intitulée Déconnectezvous au musée. Pour vous, la place du numérique au musée sera-t-elle différente?

PG: Avec comme slogan «Déconnectez-vous au musée», je voulais proposer il y a quelques années, une réflexion autour du numérique après plus de 20 ans de développement. J'aime à rappeler que l'Ocim a organisé en partenariat avec la Société des musées du Québec et Musées et Société en Wallonie, les rencontres francophones Nouvelles technologies et institutions muséales en 1998, 1999 et 2000. Ces rencontres avaient fait date dans le développement des nouvelles technologies de l'information et de la communication dans les musées, à une période où le numérique balbutiait. Le temps est venu d'avoir une nouvelle réflexion iconoclaste...

Dans cette période de confinement que nous venons de subir, le télétravail s'est développé à l'extrême, nous avons été constamment devant des écrans. Dans les loisirs, dans notre vie quotidienne, nous sommes en situation de confrontation quasi-perpétuelle aux écrans. Nous, notre public, sommes dans un état de saturation qui fait que nous recherchons des lieux libres d'écrans. Le mot «écran » exprime bien ce qu'il veut dire : une barrière, un isolant entre une chose et une autre. Or, ce que l'on recherche quand on vient dans un musée, c'est vraiment être en relation directe avec l'objet: pour que naissent l'émotion, et de là une connaissance. Quand nous sommes devant un vrai fossile - devant les restes d'un être qui a réellement existé, comme un crâne de Néandertalien - c'est tout à fait différent que d'être devant un simple moulage ou une représentation sur un écran. L'émotion est clairement tangible.

Je suis un peu atterré par cette course effrénée au numérique, je crois qu'il faut vraiment faire attention. N'oublions pas le triptyque fondateur du musée : l'objet réel, le médiateur humain et le public réel. Je crois que c'est vraiment le cœur du musée. Cependant, je ne voudrais pas apparaître comme rétrograde, et la réplique est facile quand on est dans un muséum, car le numérique est avant tout un formidable outil à notre service. Au muséum de Nantes, nous avons en charge la gestion d'environ 1,5 millions d'objets ou de spécimens: on ne peut pas avoir 1,5 millions d'objets ou spécimens en exposition permanente, 1 à $2 \%$ tout au plus. Si l'on veut rendre compte de l'ensemble des collections aux publics - qu'ils soient amateurs, scientifiques ou simplement curieux - la mise à disposition ne peut se faire que par les moyens du numérique. La réalité augmentée est aussi une manière extraordinaire d\&apos;enrichir notre rapport à l'objet et, je pense ici particulièrement aux fossiles en paléontologie que le public a bien du mal à se représenter.

Sur ce projet de Déconnectez-vous au musée, il faut que nous allions plus loin en l'affirmant d'emblée à nos visiteurs. Nous allons avancer sur cette question dans notre prochain PSC.

$\boldsymbol{s c}$ : Au muséum de Nantes, la réouverture va se faire de quelle manière?

PG : La réouverture est prévue le 8 août. C'est le jour du démarrage du Voyage à Nantes, un grand moment orienté art contemporain-art dans la rue, mais aussi un événement sur lequel le muséum est attendu. Notre personnel est impatient de retrouver le public! Nous rouvrons presque deux mois après les autres musées en raison de travaux concernant l'accueil, préfiguration d'une rénovation globale de l'établissement dans un style vernien (et donc nantais) que l'on espère voir se terminer dans ce mandat.

$\boldsymbol{s c}$ : Quels sont les changements à court et moyen termes?

PG : II est clair que les enjeux environnementaux seront renforcés. Mais les changements que nous allons avoir seront surtout d'ordre économique, et par conséquent social. Si pour cette année encore les budgets des muséums - et d'une manière générale ceux des établissements de CSTI - sont à peu près maintenus, ce ne sera pas le cas en 2021 car les collectivités locales vont subir des pertes en recettes très importantes. Des choix seront faits et je pense que pour les plus fragiles d'entre nous, ce sera de l'ordre de la survie (voire 
de la disparition). C'est à nous d'être inventifs dans nos productions, dans nos engagements et dans nos solidarités!

\section{NOTES}

1. Philippe Guillet est membre du CA de l'Amcsti (président de l'Amcsti de 1996 à 2000, puis de 2012 à 2017). Il a également dirigé l'Ocim (de 1988 à 2007) et fut secrétaire général d'Icom-France (de 2007 à 2010), puis vice-président (de 2010 à 2013).

\section{RÉSUMÉS}

Le rôle des musées de sciences est plus que jamais engagé et la sortie de confinement réclame un renouveau des pratiques. Dans cet entretien, le directeur du muséum de Nantes s'interroge : dans quelles mesures nos institutions ont-elles réellement les moyens de se ré-imaginer, sachant que leurs actions et leur gestion s'inscrivent dans des politiques locales?

\section{INDEX}

Mots-clés : gestion fonctionnelle (organisation); politique culturelle ; politique de public ; centre de sciences, CCSTI; musée scientifique, technique et industriel

\section{AUTEURS}

\section{PHILIPPE GUILLET}

Directeur du muséum de Nantes

philippe.guillet@nantesmetropole.fr

\section{SAMUEL CORDIER}

Directeur de l'Ocim

samuel.cordier@u-bourgogne.fr 